\title{
Expounding the influence of chemicals and processing techniques on the shelf life of Fig (Ficus carica L.)
}

\author{
Anwaar Ahmed ${ }^{\mathrm{a}}$, Muhammad Nadeem ${ }^{\mathrm{b}}$, Rai Muhammad Amir ${ }^{\mathrm{a}}$, Muhammad Sameem Javed ${ }^{\mathrm{c}}$, \\ Adnan Amjad ${ }^{\mathrm{c}}$, Shahzad Saleem ${ }^{\mathrm{d}}$, Asim Mehmood ${ }^{\mathrm{d}}$, Muhammad Asif Khan ${ }^{\mathrm{e}}$, Kashif Ameer ${ }^{\mathrm{a}}$, \\ Ashir Masroor $^{\mathrm{e}}$, Muhammad Imran ${ }^{\mathrm{f}}$, Muhammad Wasim Sajid ${ }^{\mathrm{d}, *}$ \\ ${ }^{a}$ Institute of Food and Nutritional Sciences, PMAS-Arid Agriculture University, Rawalpindi-Pakistan \\ b Department of Environmental Sciences, COMSATS University Islamabad, Sahiwal-Pakistan, \\ Vehari-Pakistan \\ c Institute of Food Science and Nutrition, Bahauddin Zakariya University, Multan-Pakistan \\ ${ }^{d}$ Department of BioSciences, COMSATS University Islamabad, Sahiwal-Pakistan \\ e UAF, Sub-Campus Burewala-Vehari, Pakistan \\ f Faculty of Allied Health Sciences, The University of Lahore, Lahore, Pakistan
}

${ }^{*}$ Corresponding author, e-mail: muhammad.wasim@cuisahiwal.edu.pk

\begin{abstract}
The quality of dried fig was compared by treating blanched figs with potassium metabisulfite, NaCl, ascorbic acid (AA) at various concentrations (2, 3, 4\%) and sucrose solution (30, 40, 50\%). In comparison of results for functional attributes, $T_{5}$ (blanching $+4 \%$ AA dipped for $10 \mathrm{~min}$ ) stood prominent in retaining higher ascorbic acid, phenolics and antioxidant activity followed by $T_{4}$ (blanching $+3 \%$ AA dipped for $10 \mathrm{~min}$ ) and $T_{3}$ (blanching $+2 \%$ AA dipped for $10 \mathrm{~min}$ ). However, higher fibers and total sugars were found in $T_{8}$ (blanching $+50 \%$ sucrose dipped for $30 \mathrm{~min}$ ) and $T_{7}$ (blanching $+40 \%$ sucrose dipped for $30 \mathrm{~min}$ ). Eventually, sensory evaluation of the stored samples demonstrated best scores for the osmotically dehydrated fig with 30 and $40 \%$ sucrose prior to cabinet drying. Overall, the results of present investigation have significant contribution to the food industry. Of note the shelf life and stability of fig could be enhanced upon bringing the result of this finding in practice.
\end{abstract}

KEYWORDS: dried fig, chemical pre-treatment, nutritional attributes, functional properties, sensory evaluation

\section{INTRODUCTION}

Fig (Ficus carica L.) is a commonly grown food crop in semi-arid climate of the eastern Mediterranean region and southwest Asia ${ }^{1}$. The annual production of fig is about one million metric tons in the world with a cultivated area of $419000 \mathrm{ha}^{2}$. It is a delicious fruit with highly nutritional and functional attributes. A serving of $100 \mathrm{~g}$ fig daily provides the mentioned nutritional information: $6.2 \%$ riboflavin, $7.1 \%$ thiamin, $30 \%$ iron, and $15.8 \%$ calcium $^{3}$. Among all fruits, dried fig is a rich source of $\mathrm{Cu}, \mathrm{K}$, $\mathrm{Mn}, \mathrm{Mg}$, vitamins, dietary fibers and amino acids ${ }^{4,5}$. Dried fig contains the highest amount of crude fiber and phytochemicals. Different varieties of fig have numerous carotenoids like $\alpha$-carotene, cryptoxanthin, lycopene and lutein, with the lycopene in larger quantity than lutein and $\alpha$-carotene ${ }^{6-8}$. Keeping in view the compositional strength of fig, its extract is widely used for medicinal purposes ${ }^{3,8,9}$. Epidemiological studies have shown that consump- tion of fig can reduce heart disease, cancer and other degenerative diseases ${ }^{6,10,11}$.

Unfortunately, the quality and characteristics of fig are mostly influenced by the high amount of sugars and organic acids. Fresh fruits of fig are perishable and have a very short postharvest life ${ }^{10}$. It deteriorates due to fermentation and other physical factors that reduce the marketing life and storage period. Better quality dehydrated products can be achieved by osmotic dehydration process and or its combination with other drying methods. Osmotic dehydration can be done by putting the food commodity in concentrated sugar solution and partially removing the moisture from the product. Chemicals which are generally recommended as safe (GRAS) such as $\mathrm{SO}_{2}$ fumigation and use of potassium metabisulfite (KMS) and sodium carbonate are widely recommended worldwide to achieve a product of better sensory properties with increased shelf life ${ }^{12,13}$. Very few studies have 
been carried out to increase the shelf life of dried figs in Pakistan. Therefore, the present study was conducted to enhance the storage life and to assess the keeping quality of stored dried figs as affected by pretreatments during ambient storage.

\section{MATERIALS AND METHODS}

\section{Collection of samples and treatments}

The fully matured figs were harvested in District Ghizer, Gilgit Baltistan, Pakistan. Fruits were cleaned and washed to remove all adhering dirt and dust particles. The fresh fruits were divided into 9 groups to be treated with different chemicals as per following plan: The pretreatments were applied in the following scheme; all chemicals were prepared in $\%(\mathrm{w} / \mathrm{v})$ :

\begin{tabular}{ll}
\hline Treatment & Chemical \\
\hline$T_{0}:$ & control (without any treatment) \\
$T_{1}:$ & 0.15\% KMS dip for $5 \mathrm{~min}$ \\
$T_{2}:$ & blanching+2\% NaCl dip for $60 \mathrm{~min}$ \\
$T_{3}:$ & blanching+2\% AA dip for $10 \mathrm{~min}$ \\
$T_{4}:$ & blanching+3\% AA dip for $10 \mathrm{~min}$ \\
$T_{5}:$ & blanching+4\% AA dip for $10 \mathrm{~min}$ \\
$T_{6}:$ & blanching+30\% sucrose dip for $30 \mathrm{~min}$ \\
$T_{7}:$ & blanching+40\% sucrose dip for $30 \mathrm{~min}$ \\
$T_{8}:$ & blanching+50\% sucrose dip for $30 \mathrm{~min}$ \\
\hline
\end{tabular}

Further, the fruits were dehydrated using solar drier facility available at the Agriculture Department, Gilgit. The dried fruits were sealed in plastic bags and brought to the Postharvest Lab of Food Technology, PMAS, Arid Agriculture University, Rawalpindi, stored at room temperature and analyzed for their shelf stability for a period of six months.

\section{Storage and physicochemical analysis}

Moisture content, crude fiber, titratable acidity and ascorbic acid were determined by following AOAC $(2000){ }^{14}$ procedures while total sugars were determined by Lane and Eynon method ${ }^{15}$.

\section{Moisture content}

Moisture content was determined according to the official protocol of AOAC $(2000){ }^{14}$. Pre-weight samples were subjected to hot air oven at $100-105^{\circ} \mathrm{C}$ till constant weight. Then the moisture content was calculated by the following formula:

Moisture $\%=\frac{\text { Sample weight }- \text { Weight after drying }}{\text { Sample weight }} \times 100$

\section{Crude fiber}

Fat free samples were taken for estimation of crude fiber, the samples were treated with $1.25 \%(\mathrm{v} / \mathrm{v})$
$\mathrm{H}_{2} \mathrm{SO}_{4}$ solution to digest the samples and then $1.25 \%(\mathrm{v} / \mathrm{v}) \mathrm{NaOH}$ solution to neutralize the samples. The rest of filtrate was charred after drying. After that, samples were placed in muffle furnace at $550^{\circ} \mathrm{C}$ to ignite the samples and the resulted materials were weighed (AOAC, 2000). By using the following formula, we can calculate the fiber content

$$
\text { Fiber } \%=\frac{\text { Ash weight }}{\text { Sample weight }} \times 100
$$

\section{Titratable acidity}

Five grams thoroughly mixed fig pulp samples were prepared and $100 \mathrm{ml}$ volume was maintained by addition of distilled water. Thereafter, $10 \mathrm{ml}$ of the filtrate was taken and 2-3 drops of phenolphthalein indicator was added and titrated against $0.1 \mathrm{~N} \mathrm{NaOH}$ till light pink color appeared (AOAC, 2000). The acidity in percentage was calculated by following formula:

$$
\text { Acidity }(\%)=\frac{\text { Acid weight } \times \text { Base normality } \times \text { Titer }}{\text { Sample weight } \times \text { Aliquot taken }} \times 100
$$

\section{Ascorbic acid content}

Ascorbic acid was titrimetrically determined by using sodium 2,6-dichlorophenol indophenol dye (AOAC, 2000). Fig sample (10 g) was accurately weighed and grounded using mortar and pestle in $20 \mathrm{ml}$ of metaphosphoric acid - acetic acid. The mixture was further grounded and strained through muslin and the extract was made up to $100 \mathrm{ml}$ with the metaphosphoric-acetic acid mixture. Five $\mathrm{ml}$ of this acid-mixture solution was pipetted into $50 \mathrm{ml}$ Erlenmeyer flask containing $2 \mathrm{ml}$ of the extract. The samples were titrated separately with the indophenol dye until a light rose pink color was persisted for 5 seconds. The amount of dye used in the titration was determined and used for calculating vitamin C content present in the sample.

\section{Total sugars}

Amounts of total sugars present in fig were determined by using the methods adopted by Lane and Enyon ${ }^{15}$. The amounts of reducing and nonreducing sugars were measured (AOAC, 2000).

\section{Total phenolic content and antioxidant activity}

200 grams of fig sample were taken in $500 \mathrm{ml}$ flask, then filled with methanol. The sample flask was placed in orbital shaker incubator for $48 \mathrm{~h}$ at ambient temperature. The resultant samples were filtered, thereafter, the extract was concentrated 
by using rotary evaporator till the sample amount was reduced to $1 \mathrm{ml}$. The solvent was further removed by subjecting sample to the purified gentle stream of $\mathrm{N}_{2}$ gas. The sample was stored at $-4{ }^{\circ} \mathrm{C}$ till further analysis ${ }^{16}$. Total phenolic contents were measured using the Folin-Ciocalteu reagent in UV-Vis spectrophotometer ${ }^{17}$. Gallic acid was used as standard and the standard curve (obtained by running different concentrations of gallic acid) was used to calculate the total phenolic contents in the sample. The absorbance of the samples was measured at $765 \mathrm{~nm}$. The results were expressed as mg GAE/100 g.

Antioxidant activity was measured by the method as described ${ }^{18}$, that involves the use of the free radical 2,2-diphenyl-1-picrylhydrazyl (DPPH). The absorbance of the samples was spectrophotometrically measured at $517 \mathrm{~nm}$. Antioxidant activity was calculated as \% inhibition of DPPH radical by the following formula:

$$
\% \text { Inhibition }=\frac{\mathrm{A}_{\text {blank }}-\mathrm{A}_{\text {sample }}}{\mathrm{A}_{\text {blank }}} \times 100
$$

\section{Sensory evaluation}

The sensory evaluation of the dried fig fruit (color, flavor, taste and overall acceptability) was carried out (at one month interval) by a panel of five trained judges using 9-point hedonic scale rating method following the method of Ref. 19.

\section{Statistical analysis}

Data obtained for each parameter was analyzed to check the level of significance $(p \leqslant 0.05)$ and means were compared by Duncan Multiple Range Test (DMRT) according to Steel et $\mathrm{al}^{20}$ using MSTATC software ${ }^{21}$.

\section{RESULTS AND DISCUSSION}

\section{Physicochemical analysis}

The mean values for moisture content of pretreated dried fig ranged from 11.41-16.03\% (Table 1). Higher moisture content was observed in control sample $\left(T_{0}, 16.03 \%\right)$, while lowest moisture content was observed in the sample treated with $40 \%$ sucrose solution $\left(T_{8}, 11.71 \%\right)$. It was found that the moisture content of dried figs differed significantly $(p<0.05)$ among the treatments. A significant decrease in moisture was observed in the samples treated with sugar solution, when compared with other chemically treated figs. The samples treated with 2,3 , and $4 \%$ concentrations of ascorbic acid and KMS solution showed a non-significant difference among each other; however, differed significantly from other fig samples treated with sugar, $\mathrm{NaCl}$ and sucrose solution.There was a decreasing trend in the moisture content of pretreated dried fig samples during storage of six months. The mean values for moisture content during storage intervals ranged from $13.50-14.84 \%$. Control samples carried highest moisture (16.03\%) but the samples treated with $50 \%$ sucrose showed minimum moisture $(11.41 \%)$ while the rest of samples responded variedly in between these values towards the end of storage. The fresh fig contained $75-80 \%$ moisture and after sun drying moisture reduced to $14-15 \%$. However, during storage of six months at ambient temperature moisture also decreased and reached the level of $13.50 \%$. The moisture content present in fig samples was less than that reported by earlier studies $^{8,22}$. During the present study, moisture content of the pretreated dried fig remained in the lower limits which might be due to variation in storage conditions, drying technique, temperature and humidity during storage of the final product.

Crude fiber of pretreated dried fig samples ranged from 3.29 to $4.10 \%$ as can be seen in Table 2 . Higher crude fiber was found in $T_{8}(4.10 \%)$ while the lowest was observed in control $(3.29 \%)$. The increase in fiber content was observed due to increase in the pretreatment concentration. The data showed that there was a significant decreasing trend on crude fiber during storage of dried figs. Among all, fig fruits either in dried or fresh form contain large amount of fiber content which facilitate the digestive system and remove ingestion problems ${ }^{9}$. The conclusion of our recent observations is aligned with the results of Ramulu ${ }^{23}$, who reported the same findings related to dietary fiber of fig fruit.

The data pertaining to total sugars shown in Table 3 indicated increasing trend of total sugars during storage against all treatments. The mean values for total sugar among different treatments ranged from $24.33-43.84 \mathrm{~g} / 100 \mathrm{~g}$. Significantly $(p<0.05)$, higher total sugar contents were observed in $T_{6}, T_{7}$, and $T_{8}$, respectively. Higher total sugar contents in $T_{8}, T_{7}$, and $T_{6}$ might be due to increase in the concentration of sucrose solution during pretreatment. Similarly, non-significant results were found for $\mathrm{NaCl}$ and ascorbic acid treated fruit along with control set. During storage of dried fruits, complex carbohydrates are converted into simple sugars and loss of moisture augments ultimate increase in soluble sugar concentration ${ }^{10,24}$. Naikwadi, Pawar, and Thota ${ }^{10,25,26}$ also reported 
Table 1 Effect of different treatments on moisture content in dried fig during ambient storage.

\begin{tabular}{|c|c|c|c|c|c|c|c|c|}
\hline \multirow{2}{*}{ Treat } & \multicolumn{7}{|c|}{ Storage period (days) } & \multirow{2}{*}{ Mean \pm SD } \\
\hline & 0 & 30 & 60 & 90 & 120 & 150 & 180 & \\
\hline & $00 \pm$ & $10.50 I$ & & & & 15.6 & & \\
\hline$T_{1}$ & & & & & . & 10.0 & & 10. \\
\hline$T_{2}$ & 14.8 & 14.40 & $0^{\mathrm{f}-\mathrm{n}}$ & $14.10 \pm$ & $13.80 \pm$ & $13.60=$ & 12. & $3^{c}$ \\
\hline$T_{3}$ & $\pm 0.80^{\mathrm{b}-\mathrm{f}}$ & $15.67 \pm$ & $15.30 \pm 0.80^{\mathrm{b}-\mathrm{h}}$ & $15.10 \pm 0.60^{\mathrm{c}-\mathrm{i}}$ & $14.90 \pm 0.90^{\mathrm{c}-\mathrm{k}}$ & $14.70 \pm$ & 14. & $0.44^{\mathrm{b}}$ \\
\hline$T_{4}$ & 1 & 1 & $15.20 \pm$ & $15.00 \pm 1.00^{\mathrm{cj}}$ & $14.80 \pm$ & $14.70 \pm$ & 14.5 & ${ }^{1} 15$ \\
\hline$T_{5}$ & $15.37 \pm 0.42^{\mathrm{b}-\mathrm{g}}$ & $15.50 \pm 1.10^{\mathrm{b}-\mathrm{f}}$ & $15.30 \pm 1.30^{\mathrm{b}-\mathrm{h}}$ & $15.10 \pm 0.80^{\mathrm{c}-\mathrm{i}}$ & $14.90 \pm 1.30^{\mathrm{c}-\mathrm{k}}$ & $14.80 \pm 0.90^{\mathrm{c}-\mathrm{k}}$ & $14.27=$ & $=0.42^{\mathrm{b}}$ \\
\hline & $14.00 \pm 1.20^{\mathrm{h}-\mathrm{p}}$ & $13.70 \pm 1.40^{\mathrm{j}-\mathrm{q}}$ & $13.60 \pm 0.90^{\mathrm{k}-\mathrm{q}}$ & $13.40 \pm 0.40^{1-q}$ & $13.20 \pm 0.80^{\mathrm{m}-\mathrm{r}}$ & & & \\
\hline$T_{7}$ & 1 & $13.20 \pm 0.70^{\mathrm{m}-\mathrm{r}}$ & $13.00 \pm 0.60^{\mathrm{n}-\mathrm{s}}$ & $12.90 \pm$ & $12.70 \pm$ & $12.60 \pm$ & ${ }^{t} 12.00 \pm 0$ & 12. \\
\hline & $12.00 \pm$ & $11.80 \pm 0.90^{\text {stu }}$ & $11.60 \pm 0.80^{\text {tu }}$ & $13.60 \pm 0.70^{\mathrm{k}-\mathrm{q}}$ & $11.20 \pm 0.80^{\mathrm{u}}$ & $11.00 \pm 0.70^{\mathrm{u}}$ & $10.80 \pm 0.40^{\mathrm{u}}$ & $11.71 \pm 0.94^{f}$ \\
\hline & $14.84 \pm 1.5$ & $14.66 \pm 1.51^{\mathrm{a}}$ & 浮 & $14.53 \pm 1.06^{\mathrm{a}}$ & $14.04 \pm 1.45^{\mathrm{bc}}$ & $13.87 \pm 1.44^{\mathrm{cd}}$ & $13.50 \pm 1.48^{\mathrm{d}}$ & \\
\hline
\end{tabular}

LSD: $\mathrm{T}=0.52, \mathrm{~S}=0.45$, TS $=1.36$. Values are means of triplicate. The means within the row bearing same letter(s) are statistically significant at $p<0.05$.

Table 2 Effect of different treatments on fiber in dried fig during ambient storage.

\begin{tabular}{|c|c|c|c|c|c|c|c|c|}
\hline \multirow{2}{*}{ Treat } & \multicolumn{7}{|c|}{ Storage period (days) } & \multirow{2}{*}{ Mean \pm SD } \\
\hline & 0 & 30 & 60 & 90 & 120 & 150 & 180 & \\
\hline$T_{0}$ & $4.00 \pm 0.40^{\mathrm{a}-\mathrm{d}}$ & $3.80 \pm 0.70^{\mathrm{a}-\mathrm{e}}$ & $3.60 \pm 0.80^{b-f}$ & $3.20 \pm 0.20^{\mathrm{d}-\mathrm{g}}$ & $3.00 \pm 0.30^{\text {efg }}$ & $2.80 \pm 0.30^{f g}$ & $2.60 \pm 0.40^{g}$ & $3.29 \pm 0.53^{\mathrm{d}}$ \\
\hline $\begin{array}{l}I_{0} \\
T_{1}\end{array}$ & $4.00 \pm 1.00^{\mathrm{a}-\mathrm{d}}$ & $3.80 \pm 0.80^{\mathrm{a}-\mathrm{e}}$ & $3.60 \pm 0.40^{b-f}$ & $3.73 \pm 0.31^{\mathrm{a}-\mathrm{e}}$ & $3.40 \pm 0.30^{\mathrm{c}-\mathrm{g}}$ & $3.20 \pm 0.40^{\mathrm{d}-\mathrm{g}}$ & $3.00 \pm 0.04^{\text {efg }}$ & $3.53 \pm 0.35^{\mathrm{bc}}$ \\
\hline$T_{2}$ & $4.20 \pm 0.30^{\mathrm{abc}}$ & $4.00 \pm 0.70^{\mathrm{a}-\mathrm{d}}$ & $3.80 \pm 0.80^{\mathrm{a}-\mathrm{e}}$ & $3.60 \pm 0.60^{\mathrm{b}-\mathrm{f}}$ & $3.40 \pm 0.50^{c-g}$ & $3.40 \pm 0.50^{\mathrm{c}-\mathrm{g}}$ & $3.20 \pm 0.20^{\mathrm{d}-\mathrm{g}}$ & $3.66 \pm 0.36^{b c}$ \\
\hline$T_{3}$ & $4.00 \pm 0.40^{\mathrm{a}-\mathrm{d}}$ & $3.70 \pm 0.46^{\mathrm{a}-\mathrm{e}}$ & $3.60 \pm 0.40^{\mathrm{b}-\mathrm{f}}$ & $3.40 \pm 0.25^{c-g}$ & $3.40 \pm 0.60^{c-g}$ & $3.20 \pm 0.60^{\mathrm{d}-\mathrm{g}}$ & $3.20 \pm 0.10^{\mathrm{d}-\mathrm{g}}$ & $3.50 \pm 0.29^{b c}$ \\
\hline$T_{4}$ & $4.20 \pm 0.50^{\mathrm{abc}}$ & $3.53 \pm 0.40^{\mathrm{c}-\mathrm{f}}$ & $4.08 \pm 0.38^{a b c}$ & $3.80 \pm 0.70^{\mathrm{a}-\mathrm{e}}$ & $3.80 \pm 0.70^{\mathrm{a}-\mathrm{e}}$ & $3.60 \pm 0.70^{\mathrm{b}-\mathrm{f}}$ & $3.07 \pm 0.29^{\mathrm{efg}}$ & $3.73 \pm 0.38^{b c}$ \\
\hline$T_{5}$ & $4.00 \pm 0.30^{\mathrm{a}-\mathrm{d}}$ & $4.00 \pm 0.92^{\mathrm{a}-\mathrm{d}}$ & $3.60 \pm 0.50^{b-f}$ & $3.40 \pm 0.35^{\mathrm{c}-\mathrm{g}}$ & $3.20 \pm 0.30^{\mathrm{d}-\mathrm{g}}$ & $3.00 \pm 0.50^{\mathrm{efg}}$ & $3.00 \pm 0.60^{\mathrm{efg}}$ & $3.46 \pm 0.43^{\mathrm{cd}}$ \\
\hline$T_{6}$ & $4.00 \pm 0.20^{\mathrm{a}-\mathrm{d}}$ & $3.80 \pm 0.50^{\mathrm{a}-\mathrm{e}}$ & $3.60 \pm 0.50^{b-f}$ & $3.40 \pm 0.50^{c-g}$ & $3.20 \pm 0.40^{\mathrm{d}-\mathrm{g}}$ & $3.00 \pm 0.40^{\text {efg }}$ & $3.00 \pm 0.02^{\text {efg }}$ & $3.43 \pm 0.39^{\mathrm{cd}}$ \\
\hline$T_{7}$ & $4.20 \pm 0.50^{\mathrm{abc}}$ & $4.00 \pm 0.40^{\mathrm{a}-\mathrm{d}}$ & $3.80 \pm 0.60^{\mathrm{a}-\mathrm{e}}$ & $3.80 \pm 0.45^{\mathrm{a}-\mathrm{e}}$ & $3.60 \pm 0.60^{b-f}$ & $3.60 \pm 0.60^{b-f}$ & $3.40 \pm 0.03^{c-g}$ & $3.77 \pm 0.27^{b}$ \\
\hline$T_{8}$ & $4.50 \pm 0.50^{a}$ & $4.40 \pm 0.50^{\mathrm{ab}}$ & $4.20 \pm 0.30^{a b c}$ & $4.20 \pm 0.60^{\mathrm{abc}}$ & $4.00 \pm 0.50^{\mathrm{a}-\mathrm{d}}$ & $3.80 \pm 0.70^{\mathrm{a}-\mathrm{e}}$ & $3.60 \pm 0.50^{\mathrm{b}-\mathrm{f}}$ & $4.10 \pm 0.32^{\mathrm{a}}$ \\
\hline Mean & $4.12 \pm 0.17^{a}$ & $3.89 \pm 0.25^{\mathrm{ab}}$ & $3.76 \pm 0.23^{b c}$ & $3.61 \pm 0.30^{\mathrm{cd}}$ & $3.44 \pm 0.31^{\text {de }}$ & $3.29 \pm 0.33^{\text {ef }}$ & $3.19 \pm 0.28^{\mathrm{f}}$ & \\
\hline
\end{tabular}

LSD: $\mathrm{T}=0.16, \mathrm{~S}=0.14, \mathrm{TS}=0.82$. Values are means of triplicate. The means within the row bearing same letter(s) are statistically significant at $p<0.05$.

the similar results related to our findings that during drying and storage the total sugar of fig fruit increased.

Titratable acidity of dried fig samples ranged from $0.08-0.152 \%$ and an overall increasing trend was witnessed in all treatments (Table 4). Significantly $(p<0.05)$ higher value was found in $T_{5}$ followed by $T_{4}$ and $T_{3}$, while the lowest values with non-significant pattern were found in other treatments and control sample. During extended storage, moisture losses and sugar fermentation occurs that resulted in increased concentration of organic acids. Similarly, the increase in titratable acidity was also related to increase in ascorbic acid concentration in the pretreatment. Our findings are in agreement with Naikwadi and Pawar ${ }^{10,26}$, who found a gradual increase in titratable acidity of dried fig during storage.

Storage behavior of ascorbic acid (AA) in dried figs showed a declining trend in all treatments (Table 5). The mean values among different treatments ranged from $1.73-2.75 \mathrm{mg} / 100 \mathrm{~g}$. The

Table 3 Effect of different treatments on total sugars in dried fig during ambient storage

\begin{tabular}{|c|c|c|c|c|c|c|c|}
\hline \multirow{2}{*}{ Treat } & \multicolumn{6}{|c|}{ Storage period (days) } & \multirow{2}{*}{ Mean \pm S.D. } \\
\hline & 0 & 30 & 90 & 120 & 150 & 180 & \\
\hline$T_{0}$ & $22.60 \pm 1.20^{\mathrm{u}}$ & $23.80 \pm 0.90^{r-1}$ & 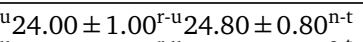 & $24.50 \pm 0.70^{\mathrm{o}-\mathrm{u}}$ & $24.40 \pm 0.90^{\mathrm{p}-\mathrm{u}}$ & $26.20 \pm 0.80^{\text {nop }}$ & $24.33 \pm 1.09^{\mathrm{d}}$ \\
\hline$T_{1}$ & $3.40 \pm 1.30^{\text {tu }}$ & $23.80 \pm 1.00^{\mathrm{r}-\mathrm{c}}$ & ${ }^{\mathrm{u}} 23.80 \pm 0.90^{\mathrm{r}-\mathrm{u}} 24.60 \pm 0.90^{\mathrm{o}-\mathrm{t}}$ & $25.40 \pm 0.52^{\mathrm{n}-\mathrm{s}}$ & $25.10 \pm 1.00^{\mathrm{n}-\mathrm{t}}$ & $26.40 \pm 1.20^{\text {no }}$ & $24.64 \pm 1.07^{\mathrm{d}}$ \\
\hline$T_{2}$ & $23.40 \pm 1.20^{\text {tu }}$ & $24.00 \pm 1.10^{r-1}$ & $\mathrm{u}_{24.20} \pm 0.80^{\mathrm{q}-\mathrm{u}} 24.20 \pm 0.70^{\mathrm{q}-\mathrm{u}}$ & $24.20 \pm 1.00^{\mathrm{q}-\mathrm{u}}$ & $25.00 \pm 0.70^{\mathrm{n}-\mathrm{t}}$ & $26.40 \pm 1.30^{\text {no }}$ & $24.47 \pm 0.97^{d}$ \\
\hline$T_{3}$ & $23.60 \pm 0.80^{\text {st }}$ & $24.00 \pm 1.20^{\mathrm{r}-\mathrm{l}}$ & ${ }^{\mathrm{u}} 24.40 \pm 1.20^{\mathrm{p}-\mathrm{u}} 24.60 \pm 1.00^{\mathrm{o}-\mathrm{t}}$ & $25.00 \pm 1.20^{\mathrm{n}-\mathrm{t}}$ & $25.20 \pm 0.80^{\mathrm{n}-\mathrm{t}}$ & $26.00 \pm 1.20^{\mathrm{n}-\mathrm{q}}$ & $24.69 \pm 0.80^{\mathrm{d}}$ \\
\hline$T_{4}$ & $23.60 \pm 0.90^{\text {st }}$ & $23.80 \pm 1.30^{\mathrm{r}-\mathrm{t}}$ & ${ }^{\mathrm{u}} 24.60 \pm 1.30^{\mathrm{o}-\mathrm{t}} 25.20 \pm 1.30^{\mathrm{n}-\mathrm{t}}$ & $25.40 \pm 0.90^{\mathrm{n}-\mathrm{s}}$ & $25.30 \pm 1.10^{\mathrm{n}-\mathrm{t}}$ & $26.20 \pm 0.85^{\text {nop }}$ & $24.87 \pm 0.93^{d}$ \\
\hline$T_{5}^{4}$ & $24.20 \pm 0.90^{\mathrm{q}-}$ & $24.40 \pm 0.80^{\mathrm{p}-}$ & ${ }^{-\mathrm{u}} 24.60 \pm 1.10^{\mathrm{o}-\mathrm{t}} 24.90 \pm 0.80^{\mathrm{n}-\mathrm{t}}$ & $25.60 \pm 0.80^{\mathrm{n}-\mathrm{r}}$ & $26.60 \pm 1.20^{\mathrm{n}}$ & $26.20 \pm 1.00^{\text {nop }}$ & $24.87 \pm 0.92^{\mathrm{d}}$ \\
\hline$T_{6}$ & $32.00 \pm 0.70^{\mathrm{m}}$ & $34.60 \pm 1.40^{1}$ & $37.80 \pm 1.70^{\mathrm{jk}} 41.00 \pm 1.50^{\mathrm{hi}}$ & $43.60 \pm 1.60^{\mathrm{fg}}$ & $47.00 \pm 1.60^{\mathrm{cd}}$ & $50.00 \pm 1.50^{\mathrm{ab}}$ & $40.86 \pm 6.53^{c}$ \\
\hline$T_{7}$ & $34.60 \pm 1.40^{1}$ & $36.60 \pm 1.50^{\mathrm{jk}}$ & $40.60 \pm 1.80^{\mathrm{i}} \quad 42.80 \pm 1.40^{\mathrm{fgh}}$ & $45.80 \pm 1.70^{\mathrm{de}}$ & $48.20 \pm 1.40^{\mathrm{bc}}$ & $51.00 \pm 1.60^{\mathrm{a}}$ & $42.80 \pm 6.00^{\mathrm{b}}$ \\
\hline$T_{8}^{\prime}$ & $36.00 \pm 1.50^{\mathrm{kl}}$ & $38.00 \pm 1.40^{\mathrm{j}}$ & $41.80 \pm 1.40^{\mathrm{ghi}} 44.00 \pm 1.30^{\mathrm{ef}}$ & $46.40 \pm 1.30^{\mathrm{cd}}$ & $49.40 \pm 1.50^{\mathrm{ab}}$ & $51.30 \pm 1.40^{\mathrm{a}}$ & $43.84 \pm 5.67^{\mathrm{a}}$ \\
\hline Mean & $27.00 \pm 5.50^{g}$ & $28.09 \pm 6.29^{f}$ & $29.51 \pm 7.99^{\mathrm{e}} \quad 30.64 \pm 9.00^{\mathrm{d}}$ & $31.69 \pm 10.22^{c}$ & $32.80 \pm 11.57^{b}$ & $34.46 \pm 12.24^{\mathrm{a}}$ & \\
\hline
\end{tabular}

LSD: $\mathrm{T}=0.72 \mathrm{~S}=0.32, \mathrm{TS}=0.91$. Values are means of triplicate. The means within the row bearing same letter(s) are statistically significant at $p<0.05$. 
Table 4 Effect of different treatments on titratable acidity in dried fig during ambient storage.

\begin{tabular}{|c|c|c|c|c|c|c|c|c|}
\hline \multirow{2}{*}{ Treat } & \multicolumn{7}{|c|}{ Storage period (days) } & \multirow{2}{*}{ Mean \pm SD } \\
\hline & 0 & 30 & 60 & 90 & 120 & 150 & 180 & \\
\hline$T_{0}$ & $0.080 \pm 0.01^{\mathrm{k}-\mathrm{p}}$ & $0.080 \pm 0.01^{\mathrm{k}-\mathrm{p}}$ & $0.067 \pm 0.03^{\text {nop }}$ & $0.068 \pm 0.03^{\mathrm{m}-\mathrm{p}}$ & $p_{0.096 \pm 0.03^{g-c}}$ & $0.090 \pm 0.01^{\mathrm{h}-\mathrm{p}}$ & P $0.090 \pm 0.02^{\mathrm{h}-\mathrm{p}}$ & $0.082 \pm 0.01^{\mathrm{e}}$ \\
\hline$T_{1}$ & $0.090 \pm 0.01^{\mathrm{h}-\mathrm{p}}$ & $0.074 \pm 0.03^{1-p}$ & $0.074 \pm 0.03^{1-p}$ & $0.090 \pm 0.04^{\mathrm{h}-\mathrm{p}}$ & $0.105 \pm 0.05^{\mathrm{e}-1}$ & $0.100 \pm 0.05^{\mathrm{f}-\mathrm{n}}$ & $0.100 \pm 0.01^{f-}$ & $.090 \pm 0.01^{\mathrm{de}}$ \\
\hline$T_{2}$ & $0.067 \pm 0.02^{1-p}$ & $0.067 \pm 0.05^{\text {nop }}$ & $0.098 \pm 0.04^{\mathrm{g}-\mathrm{o}}$ & $0.101 \pm 0.05^{\mathrm{f}-\mathrm{n}}$ & $0.103 \pm 0.06^{\mathrm{f}-\mathrm{m}}$ & ${ }^{n} 0.063 \pm 0.04^{o p}$ & $0.080 \pm 0.04^{\mathrm{k}}$ & $0.084 \pm 0.01^{\mathrm{e}}$ \\
\hline$T_{3}$ & $0.090 \pm 0.01^{\mathrm{h}-\mathrm{p}}$ & $0.074 \pm 0.03^{1-p}$ & $0.098 \pm 0.06^{f-o}$ & $0.117 \pm 0.04^{\mathrm{d}-\mathrm{j}}$ & $0.130 \pm 0.01^{\mathrm{c}-\mathrm{g}}$ & $0.150 \pm 0.02^{\text {cd }}$ & $0.140 \pm 0.04^{\text {cde }}$ & $0.114 \pm 0.03^{c}$ \\
\hline$T_{4}$ & $0.085 \pm 0.02^{\mathrm{i}-\mathrm{p}}$ & $0.100 \pm 0.01^{\mathrm{f}-\mathrm{n}}$ & $0.130 \pm 0.02^{\mathrm{c}-\mathrm{g}}$ & $0.160 \pm 0.05^{\mathrm{bc}}$ & $0.160 \pm 0.02^{\mathrm{bc}}$ & $0.147 \pm 0.04^{\mathrm{cd}}$ & $0.203 \pm 0.03^{\mathrm{a}}$ & $0.141 \pm 0.04^{\mathrm{ab}}$ \\
\hline$T_{5}^{4}$ & $0.123 \pm 0.05^{\mathrm{d}-\mathrm{h}}$ & $0.120 \pm 0.04^{\mathrm{d}-\mathrm{i}}$ & $0.140 \pm 0.03^{\text {cde }}$ & $0.133 \pm 0.03^{\mathrm{c}-\mathrm{f}}$ & $0.160 \pm 0.03^{b c}$ & $0.190 \pm 0.05^{\mathrm{ab}}$ & $0.200 \pm 0.01^{\mathrm{a}}$ & $0.152 \pm 0.04^{\mathrm{a}}$ \\
\hline$T_{6}$ & $0.123 \pm 0.03^{\mathrm{d}-\mathrm{h}}$ & $0.120 \pm 0.03^{\mathrm{d}-\mathrm{i}}$ & $0.140 \pm 0.05^{\text {cde }}$ & $0.133 \pm 0.04^{\mathrm{c}-\mathrm{f}}$ & $0.160 \pm 0.02^{b c}$ & $0.190 \pm 0.02^{\mathrm{ab}}$ & $0.200 \pm 0.04^{\mathrm{a}}$ & $0.086 \pm 0.17^{\text {de }}$ \\
\hline$T_{7}$ & $0.071 \pm 0.04^{1-p}$ & $0.088 \pm 0.02^{\mathrm{i}-\mathrm{p}}$ & $0.087 \pm 0.06^{\mathrm{i}-\mathrm{p}}$ & $0.097 \pm 0.06^{\mathrm{g}-\mathrm{o}}$ & $0.094 \pm 0.06^{\mathrm{h}-\mathrm{c}}$ & $0.130 \pm 0.03^{c-g}$ & $0.123 \pm 0.05^{\mathrm{d}-\mathrm{h}}$ & $0.099 \pm 0.02^{\mathrm{d}}$ \\
\hline$T_{8}$ & $0.084 \pm 0.04^{j-p}$ & $0.109 \pm 0.05^{\mathrm{e}-\mathrm{k}}$ & $0.140 \pm 0.04^{\text {cde }}$ & $0.140 \pm 0.04^{\text {cde }}$ & $0.147 \pm 0.04^{\mathrm{cd}}$ & $0.147 \pm 0.41^{\mathrm{cd}}$ & $0.140 \pm 0.03^{\text {cde }}$ & $0.129 \pm 0.20^{\mathrm{b}}$ \\
\hline Mean & $0.084 \pm 0.01^{\mathrm{e}}$ & $0.087 \pm 0.02^{\mathrm{e}}$ & $0.103 \pm 0.03^{\mathrm{d}}$ & $0.109 \pm 0.14^{\mathrm{cd}}$ & $0.119 \pm 0.04^{b c}$ & $0.124 \pm 0.18^{\mathrm{ab}}$ & $0.133 \pm 0.05^{\mathrm{a}}$ & \\
\hline
\end{tabular}

LSD: $\mathrm{T}=0.05, \mathrm{~S}=0.04$, TS $=0.13$. Values are means of triplicate. The means within the row bearing same letter(s) are statistically significant at $p<0.05$.

Table 5 Effect of different treatments on ascorbic acid content in dried fig during ambient storage.

\begin{tabular}{|c|c|c|c|c|c|c|c|c|}
\hline \multirow{2}{*}{ Treat } & \multicolumn{7}{|c|}{ Storage period (days) } & \multirow{2}{*}{ Mean \pm SD } \\
\hline & 0 & 30 & 60 & 90 & 120 & 150 & 180 & \\
\hline & $\pm 0.40^{a-i}$ & $30^{\mathrm{a}-\mathrm{i}}$ & $20 \pm 0.20^{b-j}$ & $2.00 \pm 0.20^{\mathrm{d}-\mathrm{j}}$ & $50 \pm 0.40^{\mathrm{i}-1}$ & $1.00 \pm 0.05^{\mathrm{kl}}$ & $.30^{1}$ & $\overline{0.71^{\mathrm{d}}}$ \\
\hline$T_{1}$ & $7 \pm 0.7$ & $50 \pm 0.36^{\mathrm{a}-\mathrm{g}}$ & $2.30 \pm 0.23^{\mathrm{b}-\mathrm{j}}$ & $2.17 \pm 0.58^{\mathrm{b}-\mathrm{j}}$ & $1.97 \pm 0.58^{\mathrm{d}-\mathrm{k}}$ & $1.67 \pm 0.3$ & 1.67 & 2.1 \\
\hline$T_{1}$ & $a-g$ & 2.20 & $20 \pm 0.24^{b-j}$ & $2.00 \pm$ & $1.70 \pm$ & $1.60 \pm$ & jkl & .42 \\
\hline$T_{3}$ & $7 \pm 0.83^{\mathrm{abc}}$ & $2.77 \pm 0.67^{\mathrm{a}-\mathrm{e}}$ & $.67 \pm 0.56^{\mathrm{a}-\mathrm{f}}$ & $2.53 \pm 0.70^{\mathrm{a}-\mathrm{h}}$ & $2.33 \pm 0.59^{\mathrm{b}-\mathrm{j}}$ & $2.13 \pm 0.76^{\mathrm{c}-\mathrm{j}}$ & $2.00=$ & 2.5 \\
\hline$T_{4}$ & ab & 2.93 & $2.77 \pm$ & & a-i & $2.20 \pm$ & $5^{d-j}$ & $.39^{\mathrm{a}}$ \\
\hline$T_{5}$ & $3.37 \pm 1.0$ & $3.13 \pm$ & $2.93 \pm 1.05^{\mathrm{a}-\mathrm{d}}$ & $2.67 \pm$ & $2.57 \pm c$ & $2.37 \pm$ & $2.20=$ & $.42^{\mathrm{a}}$ \\
\hline$T$ & $2+0$ & $2.47 \pm c$ & $2.30 \pm 0.44^{\mathrm{b}-\mathrm{j}}$ & $2.00 \pm$ & $1.83 \pm 0.40^{\mathrm{e}-\mathrm{k}}$ & $1.63 \pm 0.50^{\mathrm{g}-\mathrm{k}}$ & $1.50=$ & \\
\hline$T_{7}$ & $2.60 \pm 0.40^{\mathrm{a}-\mathrm{g}}$ & $2.40 \pm 0.30^{\mathrm{a}-\mathrm{i}}$ & $2.20 \pm 0.20^{\mathrm{b}-\mathrm{j}}$ & $2.00 \pm 0.30^{\mathrm{d}-\mathrm{j}}$ & $1.80 \pm 0.50^{\mathrm{e}-\mathrm{k}}$ & $1.50 \pm 0.30^{\mathrm{i}-1}$ & $1.00 \pm 0.02^{\mathrm{kl}}$ & \\
\hline & $2.60 \pm 0.50^{\mathrm{a}-\mathrm{g}}$ & $2.40 \pm 0.40^{\mathrm{a}-\mathrm{i}}$ & $2.20 \pm 0.60^{b-j}$ & $2.20 \pm 0.60^{\mathrm{b}-\mathrm{j}}$ & $2.00 \pm 0.25^{\mathrm{d}-\mathrm{j}}$ & $1.80 \pm 0.60^{\mathrm{e}-\mathrm{k}}$ & $1.60 \pm 0.30^{\mathrm{h}-\mathrm{k}}$ & $2.11 \pm 0.34^{\mathrm{c}}$ \\
\hline İean & $2.87 \pm 0.3$ & $2.59 \pm 0.30^{\mathrm{ab}}$ & $2.42 \pm 0.29^{\mathrm{bc}}$ & $2.23 \pm 0.27^{\mathrm{cd}}$ & $2.01 \pm 0.35^{\mathrm{de}}$ & $1.77 \pm 0.42^{\mathrm{ef}}$ & $1.56 \pm 0.52^{\mathrm{f}}$ & \\
\hline
\end{tabular}

LSD: $\mathrm{T}=0.3, \mathrm{~S}=0.33$, TS $=0.98$. Values are means of triplicate. The means within the row bearing same letter(s) are statistically significant at $p<0.05$.

highest ascorbic acid content was observed in 4, 3, and $2 \%$ ascorbic acid treated fruits, respectively, followed by samples treated with KMS. A partially nonsignificant pattern with lower ascorbic acid values was found in sucrose treated fruit and control. It was revealed that increased ascorbic acid concentration in pretreatments resulted in the escalated ascorbic acid contents in the fig samples. The mean values for ascorbic acid during storage intervals of six month ranged from $2.87-1.56 \mathrm{mg} / 100 \mathrm{~g}$ and the losses were more than $50 \%$. The sensitive nature of ascorbic acid prones it to oxidation which depends upon storage conditions, water activity and oxygen availability ${ }^{27,28}$. Although it depicts good antioxidant activity and protects the commodity from darkening and microbial growth during drying but most of the ascorbic acid content are lost during drying and storage ${ }^{29,30}$. Our results are also in agreement with the previous findings of Mir and Garcia $^{31,32}$ who reported losses of ascorbic acid in dried apricot during storage.

The present study revealed that total phenolic contents reduced during storage regardless of treatments (Table 6). Maximum total phenolic contents were found in $T_{5}$ (146.19 mg GAE/100 g) followed by $T_{6}$ (144.86 mg GAE/100 g), while the minimum was found in $T_{8}$ (138.38 mg GAE/100 g). There were significant differences among the storage means and average losses were $8.44 \%$ as compared to the initial values. Phenolics have protective act against coronary heart diseases ${ }^{33}$ as well as play an important role in controlling the microbial contamination (FDA, 2019). Due to protective act of phenolic content against coronary heart diseases ${ }^{33}$, fig fruit is considered the best health promoting fruit. Duenas ${ }^{34}$ reported the similar result as shown in this study that total phenolic contents in fig fruit declined during drying as well as with the progression in storage.

Antioxidant activity (in terms of DPPH free radical scavenging capacity of dried fig) revealed a declining trend in all treatments during storage (Table 7). The mean values among different treatments ranged from $27-38.57 \%$. Higher antioxidant activity was observed in $T_{5}, T_{4}$, and $T_{3}$ that contained ascorbic acid 4,3 , and $2 \%$, while, lower activity was observed in control followed by treatment with sucrose. The mean values for antioxidant activity during storage reduced from $30.83-35.33 \%$ at the end of the storage period. The results showed 
Table 6 Effect of different treatments on total phenolic contents in dried fig during ambient storage.

\begin{tabular}{|c|c|c|c|c|c|c|c|c|}
\hline \multirow{2}{*}{ Treat } & \multicolumn{7}{|c|}{ Storage period (days) } & \multirow{2}{*}{ Mean \pm SD } \\
\hline & 0 & 30 & 60 & 90 & 120 & 150 & 180 & \\
\hline$T_{0}$ & $150.2 \pm 1.5^{\mathrm{abc}}$ & $147.3 \pm 1.0^{\mathrm{d}-\mathrm{g}}$ & $143.3 \pm 1.2^{\mathrm{i}-1}$ & $141.0 \pm 1.3^{\operatorname{lmn}}$ & $137.0 \pm 1.4^{\mathrm{pqr}}$ & $135.0 \pm 1.4^{\mathrm{rst}}$ & $133.0 \pm 1.5^{t}$ & $140.98 \pm 6.41^{\mathrm{c}}$ \\
\hline$T_{1}$ & $152.0 \pm 2.0^{\mathrm{a}}$ & $150.0 \pm 1.9^{\mathrm{a}-\mathrm{d}}$ & $147.0 \pm 1.5^{\text {efg }}$ & $143.0 \pm 1.7^{\mathrm{jkl}}$ & $140.0 \pm 1.8^{\mathrm{mno}}$ & $138.0 \pm 1.7^{\text {opq }}$ & $137.0 \pm 1.6^{\mathrm{pqr}}$ & $143.86 \pm 5.93^{b}$ \\
\hline$T_{2}$ & $148.1 \pm 1.8^{\mathrm{c}-\mathrm{f}}$ & $146.0 \pm 1.8^{\mathrm{f}-\mathrm{i}}$ & $144.0 \pm 1.6^{\mathrm{h}-\mathrm{k}}$ & $42.0 \pm 1.8^{\mathrm{klm}}$ & $140.0 \pm 1.7^{\mathrm{mno}}$ & $137.0 \pm 1.8^{\mathrm{pqr}}$ & $135.0 \pm 1.7^{\text {rst }}$ & $141.73 \pm 4.75^{c}$ \\
\hline$T_{3}$ & $150.0 \pm 1.4^{\mathrm{a}-\mathrm{d}}$ & $148.0 \pm 1.7^{\mathrm{c}-\mathrm{f}}$ & $146.0 \pm 1.4^{\mathrm{f}-\mathrm{i}}$ & $144.0 \pm 1.9^{\mathrm{h}-\mathrm{k}}$ & $142.0 \pm 1.2^{\mathrm{klm}}$ & $140.0 \pm 1.5^{\mathrm{mno}}$ & $138.0 \pm 1.8^{\mathrm{op}}$ & ${ }^{\mathrm{q}} 144.00 \pm 4.32^{\mathrm{b}}$ \\
\hline$T_{4}$ & $151.0 \pm 1.7^{\mathrm{ab}}$ & $149.0 \pm 1.7^{\mathrm{b}-\mathrm{e}}$ & $146.0 \pm 2.1^{\mathrm{f}-\mathrm{i}}$ & $144.0 \pm 1.6^{\mathrm{h}-\mathrm{k}}$ & $141.3 \pm 2.5^{\mathrm{k}-\mathrm{n}}$ & $141.0 \pm 1.9^{\mathrm{lmn}}$ & $138.0 \pm 1.9^{\mathrm{op}}$ & ${ }^{\mathrm{q}} 144.33 \pm 4.64^{\mathrm{b}}$ \\
\hline$T_{5}$ & $152.0 \pm 1.8^{\mathrm{a}}$ & $150.0 \pm 1.6^{\mathrm{a}-\mathrm{d}}$ & $148.0 \pm 2.2^{\mathrm{c}-\mathrm{f}}$ & $146.3 \pm 2.2^{\mathrm{e}-\mathrm{h}}$ & $144.0 \pm 1.5^{\mathrm{h}-\mathrm{k}}$ & $143.0 \pm 1.6^{\mathrm{jkl}}$ & $140.0 \pm 1.2^{\mathrm{m}}$ & ${ }^{10} 146.19 \pm 4.18^{\mathrm{a}}$ \\
\hline$T_{6}$ & $150.0 \pm 1.9^{\mathrm{a}-\mathrm{d}}$ & $149.0 \pm 1.5^{\mathrm{b}-\mathrm{e}}$ & $147.0 \pm 1.6^{\mathrm{efg}}$ & $145.0 \pm 1.5^{\mathrm{g}-\mathrm{j}}$ & $143.0 \pm 2.0^{\mathrm{jkl}}$ & $141.0 \pm 1.3^{\operatorname{lmn}}$ & $139.0 \pm 1.3^{\text {nop }}$ & p $144.86 \pm 4.10^{b}$ \\
\hline$T_{7}$ & $145.0 \pm 1.4^{g-j}$ & $144.0 \pm 1.4^{\mathrm{h}-\mathrm{k}}$ & $142.0 \pm 1.5^{\mathrm{klm}}$ & $143.3 \pm 4.2^{\mathrm{i}-1}$ & $138.0 \pm 1.3^{\mathrm{opq}}$ & $136.0 \pm 1.4^{q r s}$ & $135.0 \pm 1.0^{\mathrm{rst}}$ & $140.48 \pm 4.07^{\mathrm{d}}$ \\
\hline$T_{8}$ & $144.0 \pm 1.3^{\mathrm{h}-\mathrm{k}}$ & $142.0 \pm 1.3^{\mathrm{klm}}$ & $140.0 \pm 1.8^{\mathrm{mno}}$ & $137.7 \pm 1.4^{\mathrm{o}-\mathrm{r}}$ & $136.0 \pm 1.2^{\mathrm{qrs}}$ & $135.0 \pm 1.0^{\text {rst }}$ & $134.0 \pm 1.5^{\text {st }}$ & $138.38 \pm 3.74^{\mathrm{e}}$ \\
\hline Mean & $149.15 \pm 2.89^{\mathrm{a}}$ & $147.26 \pm 2.77^{b}$ & $144.81 \pm 2.66^{c}$ & $142.93 \pm 2.52^{\mathrm{d}}$ & $140.15 \pm 2.73^{\mathrm{e}}$ & $138.44 \pm 2.92^{\mathrm{f}}$ & $136.56 \pm 2.40^{g}$ & \\
\hline
\end{tabular}

LSD: $\mathrm{T}=1.03, \mathrm{~S}=0.91, \mathrm{TS}=2.37$. Values are means of triplicate. The means within the row bearing same letter(s) are statistically significant at $p<0.05$.

Table 7 Effect of different treatments on antioxidant activity in dried fig during ambient storage.

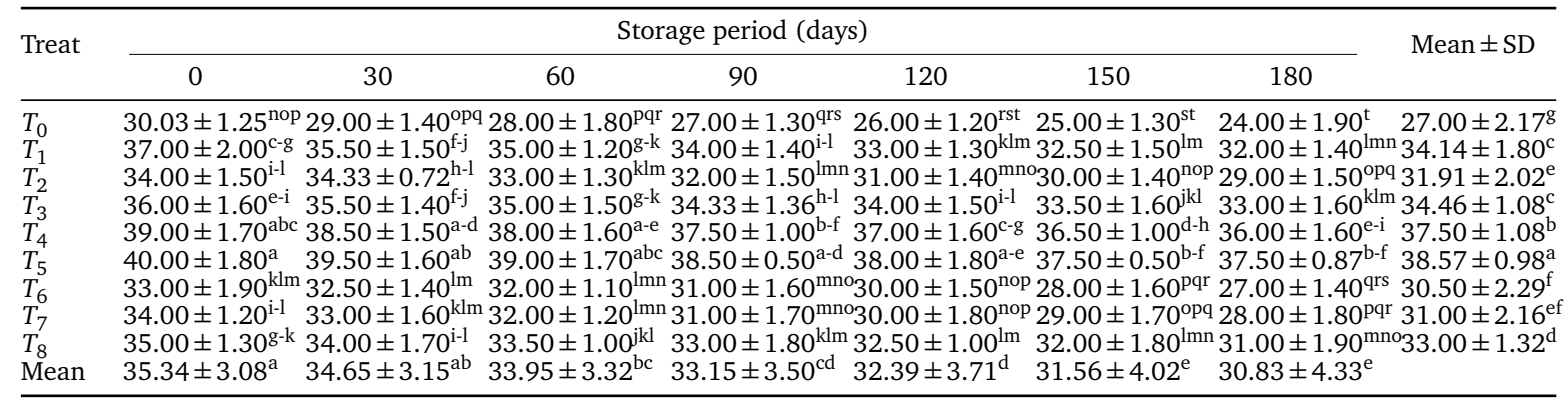

LSD: $\mathrm{T}=0.90, \mathrm{~S}=0.80, \mathrm{TS}=2.24$. Values are means of triplicate. The means within the row bearing same letter(s) are statistically significant at $p<0.05$.

a coincidence of antioxidant activity with the reducing trend of phenolic compounds and ascorbic acid during storage. It has been found that antioxidant concentration decreases during storage ${ }^{6}$. The results of present study also agreed with the findings of Patthamakanokporn and Nadheesha ${ }^{35,36}$ who reported that during storage of dried fruits the antioxidant activity decreased gradually ${ }^{37}$.

\section{Sensory evaluation}

The maximum color scores were assigned to the fruit treated with $4 \%$ ascorbic acid solution followed by those treated with KMS solution and 3\% ascorbic acid, while control set got minimum score (Table S1). The storage also has a considerable impact on the color attribute of dried fig, the initial score of 8.30 decreased to 4.93 at the termination of the experiment. A similar significant $(p<0.05)$ trend in case of flavor was also observed among different treatments (Table S2). Maximum scores were assigned to the samples treated with higher sucrose and ascorbic acid concentrations, followed by $\mathrm{NaCl}$ and KMS treated samples. The storage intervals also significantly affected the flavor score and the initial scores decreased towards the end of stor- age. The score for taste was maximum in sucrose treated figs followed by ascorbic acid treatment and a partially significant trend was found among all treatments (Table S3). The scores for taste also decreased significantly $(p<0.05)$ during storage. The scores for overall acceptability presented in Table 8, demonstrated a partially significant differences among all treatments. The maximum acceptability was retained by $4 \%$ ascorbic acid followed by $30 \%$ sucrose treated samples, while the minimum score was obtained by a set of the control. Overall acceptability was significantly affected towards the extended storage. Sensory attributes, i.e., color, flavor and taste play a significant role in determining consumer acceptability ${ }^{38}$. These characteristics are affected by different factors, i.e, final moisture content and storage environment. Studies have shown that consumer acceptance decreases with increased storage period as a result of color, flavor and taste deterioration. Among various treatments, ascorbic acid concentrations retained higher acceptability scores, while the remaining treatments showed partially similar results. It was also obvious that the extended storage resulted in poor score for the commodity. Previous research has suggested that 
Table 8 Effect of different treatments on overall acceptability (sensory properties) of dried fig during ambient storage.

\begin{tabular}{|c|c|c|c|c|c|c|c|c|}
\hline \multirow{2}{*}{ Treat } & \multicolumn{7}{|c|}{ Storage period (days) } & \multirow{2}{*}{ Mean \pm SD } \\
\hline & 0 & 30 & 60 & 90 & 120 & 150 & 180 & \\
\hline$T_{0}$ & $8.53 \pm 0.50^{\mathrm{a}}$ & $8.00 \pm 0.60^{a-d}$ & $7.00 \pm 0.50^{\mathrm{d}-\mathrm{h}}$ & $6.00 \pm 0.70^{\mathrm{h}-1}$ & $5.00 \pm 0.60^{1 \mathrm{~m}}$ & $4.00 \pm 0.60^{\mathrm{mn}}$ & $3.00 \pm 0.20^{\mathrm{n}}$ & $6.00 \pm 2.16^{\mathrm{d}}$ \\
\hline$T_{1}$ & $8.20 \pm 0.40^{\mathrm{abc}}$ & $7.40 \pm 0.40^{\mathrm{b}-\mathrm{f}}$ & $7.00 \pm 0.40^{\mathrm{d}-\mathrm{h}}$ & $6.80 \pm 0.80^{\mathrm{e}-\mathrm{i}}$ & $6.40 \pm 0.70^{f-j}$ & $6.20 \pm 0.80^{g-k}$ & $5.40 \pm 0.40^{\mathrm{jkl}}$ & $6.77 \pm 0.90^{\mathrm{bc}}$ \\
\hline$T_{2}$ & $8.20 \pm 0.40^{\mathrm{abc}}$ & $7.60 \pm 0.40^{\mathrm{b}-\mathrm{e}}$ & $7.00 \pm 0.80^{\mathrm{d}-\mathrm{h}}$ & $6.80 \pm 0.60^{\mathrm{e}-\mathrm{i}}$ & $5.60 \pm 0.60^{\mathrm{jkl}}$ & $5.20 \pm 0.60^{\mathrm{kl}}$ & $5.00 \pm 0.80^{1 \mathrm{~m}}$ & $6.49 \pm 1.24^{c}$ \\
\hline$T_{3}$ & $8.40 \pm 1.00^{\mathrm{ab}}$ & $7.60 \pm 0.70^{\mathrm{b}-\mathrm{e}}$ & $6.80 \pm 0.70^{\mathrm{e}-\mathrm{i}}$ & $6.20 \pm 0.50^{g-k}$ & $6.00 \pm 0.80^{\mathrm{h}-1}$ & $5.80 \pm 0.40^{\mathrm{i}-1}$ & $5.40 \pm 0.60^{\mathrm{jkl}}$ & $6.60 \pm 1.07^{b c}$ \\
\hline$T_{4}$ & $8.40 \pm 1.40^{\mathrm{ab}}$ & $8.00 \pm 0.80^{\mathrm{a}-\mathrm{d}}$ & $7.00 \pm 0.60^{\mathrm{d}-\mathrm{h}}$ & $6.80 \pm 0.40^{\mathrm{e}-\mathrm{i}}$ & $6.40 \pm 0.30^{f-j}$ & $6.00 \pm 0.50^{\mathrm{h}-1}$ & $5.40 \pm 0.70^{\mathrm{jkl}}$ & $6.86 \pm 1.06^{\mathrm{abc}}$ \\
\hline$T_{5}^{4}$ & $8.20 \pm 0.40^{a b c}$ & $8.00 \pm 0.60^{\mathrm{a}-\mathrm{d}}$ & $7.60 \pm 0.50^{\mathrm{b}-\mathrm{e}}$ & $7.20 \pm 0.50^{\mathrm{c}-\mathrm{g}}$ & $7.00 \pm 0.70^{\mathrm{d}-\mathrm{h}}$ & $6.40 \pm 0.50^{f-j}$ & $6.00 \pm 0.60^{\mathrm{h}-1}$ & $7.20 \pm 0.81^{\mathrm{a}}$ \\
\hline$T_{6}$ & $8.40 \pm 0.40^{\mathrm{ab}}$ & $7.60 \pm 0.70^{\mathrm{b}-\mathrm{e}}$ & $7.20 \pm 0.40^{c-g}$ & $6.80 \pm 0.40^{\mathrm{e}-\mathrm{i}}$ & $6.40 \pm 0.40^{f-j}$ & $6.20 \pm 0.70^{g-k}$ & $6.00 \pm 0.70^{\mathrm{h}-1}$ & $6.94 \pm 0.85^{\mathrm{ab}}$ \\
\hline$T_{7}$ & $8.20 \pm 1.20^{\mathrm{abc}}$ & $7.20 \pm 0.90^{c-g}$ & $7.00 \pm 0.70^{\mathrm{d}-\mathrm{h}}$ & $6.40 \pm 0.80^{\mathrm{f}-\mathrm{j}}$ & $6.20 \pm 0.50^{g-k}$ & $5.20 \pm 0.40^{\mathrm{kl}}$ & $6.00 \pm 0.90^{\mathrm{h}-1}$ & $6.60 \pm 0.97^{b c}$ \\
\hline & $8.40 \pm 1.30^{\mathrm{ab}}$ & $7.00 \pm 0.60^{\mathrm{d}-\mathrm{h}}$ & $6.80 \pm 0.60^{\mathrm{e}-\mathrm{i}}$ & $6.40 \pm 0.60^{f-j}$ & $6.00 \pm 0.40^{h-1}$ & $6.00 \pm 0.60^{\mathrm{h}-1}$ & $5.00 \pm 0.50^{1 \mathrm{~m}}$ & $6.51 \pm 1.06^{c}$ \\
\hline Mean & $8.38 \pm 0.25^{\mathrm{a}}$ & $7.60 \pm 0.36^{b}$ & $7.04 \pm 0.24^{c}$ & $6.60 \pm 0.37^{d}$ & $6.11 \pm 0.70^{\mathrm{e}}$ & $5.67 \pm 0.75^{f}$ & $5.24 \pm 0.93^{g}$ & \\
\hline
\end{tabular}

LSD: $\mathrm{T}=0.41, \mathrm{~S}=0.36, \mathrm{TS}=1.08$. Values are means of triplicate. The means within the row bearing same letter(s) are statistically significant at $p<0.05$.

pretreatment of antioxidant chemicals and additives improves storage life of dried fruits. Our results are also in agreement with Ehabe ${ }^{39}$ who established that dipping fruits prior to drying in $\mathrm{NaCl}$ and sugar solution improved quality of derived banana figs ${ }^{39}$.

\section{CONCLUSION}

The study showed a strong potential to increase the shelf life of fig by osmotic dehydration in combination with chemical treatments and drying technique. The results for sensory evaluation of the stored samples revealed that the osmotically dehydrated figs with $30-40 \%$ sucrose prior to cabinet drying were given higher scores by panelist. The results of this study can be used at industrial level to enhance shelf life stability of fig. The study results in high quality products and may also lead to substantial energy savings. Postharvest losses can also be reduced by osmotic dehydration. Initial fruit characteristics, i.e., texture, color, aroma and nutritional composition are well retained via this technique. For the development of home-scale industries and self-entrepreneurs, this technology has wider applications. The present study will provide an updated standing on osmotic dehydration and its research breach which is useful for processing industries, research purpose and academia.

\section{Appendix A. Supplementary data}

Supplementary data associated with this article can be found at http://dx.doi.org/10.2306/ scienceasia1513-1874.2019.45.547.

\section{REFERENCES}

1. Stover E, Aradhya M, Ferguson L, Crisosto CH (2007) The fig: overview of an ancient fruit. HortScience 42, $71083-71087$.

2. FAO (2015) Faostat, Agriculture Database, FAO, available at www.fao.org/faostat/en/.
3. Vinson JA, Zubik L, Bose P, Samman N, Proch J (2005) Dried fruits: excellent in vitro and in vivo antioxidants. $J$ Am Coll Nutr 24, 44-50.

4. Veberic R, Colaric M, Stampar F (2008) Phenolic acids and flavonoids of fig fruit (Ficus carica L.) in the northern Mediterranean region. Food Chemb106, 153-157.

5. Kurubar AR (2007) Studies on integrated nutrient and post harvest management of fig (Ficus carica L.), $\mathrm{PhD}$ thesis, UAS Dharwad, India.

6. Vinson JA, Proch J, Zubik L (1999) Phenol antioxidant quantity and quality in foods: Cocoa, dark chocolate, and milk chocolate. J Agric Food Chem 47, 4821-4824.

7. Su Q, Rowley KG, Itsiopoulos C, O'Dea K (2002) Identification and quantitation of major carotenoids in selected components of the Mediterranean diet: green leafy vegetables, figs and olive oil. Eur J Clin Nutr 56, 1149-1154.

8. Solomon A, Golubowicz S, Yablowicz Z, Grossman S, Bergman M, Gottlieb HE, Altman A, Kerem Z, et al (2006) Antioxidant activities and anthocyanin content of fresh fruits of common fig (Ficus carica L.). J Agric Food Chem 54, 7717-7723.

9. Lianju W, Weibin J, Kai M, Zhifeng L, Yelin W (2003) The production and research of fig (Ficus carica L.) in China. In: Corrales ML, Garcia MJB (eds) ISHS Acta Horticulturae 605: II International Symposium on Fig, Caceres, Spain, pp 191-196.

10. Naikwadi PM, Chavan UD, Pawar VD, Amarowicz R (2010) Studies on dehydration of figs using different sugar syrup treatments. J Food Sci Technol 47, 442-445.

11. Karabulut OA, Ilhan K, Arslan U, Vardar C (2009) Evaluation of the use of chlorine dioxide by fogging for decreasing postharvest decay of fig. Postharvest Biol Technol 52, 313-315.

12. Hiregoudar S, Nidoni U, Meda V, Gadade S, Patil BV (2006) A study of different drying methods for fig (Ficus carica Linn) fruit. In: ASABE/CSBE North Central Intersectional Meeting, ASABE, Michigan. 
13. Molinu MG, Venditti T, Dore A, D'Hallewin G, Serusi A, Del AC, Agabbio M (2006) Postharvest decay reduction of fig fruit (Ficus carica) by hot water sodium carbonate solutions dip. Commun Agric Appl Biol Sci 71, 923-928.

14. AOAC (2000) Official Methods of Analysis, 17th edn, The Association of Official Analytical Chemists, Gaithersburg, MD, USA. [Methods 925.10, 65.17, 974.24, 992.16].

15. Lane JH, Eynon L (1923) Methods for determination of reducing and nonreducing sugars. $J$ Sci 42, 32-37.

16. Javed MS, Randhawa MA, Butt MS, Nawaz H (2016) Effect of calcium lactate and modified atmosphere storage on biochemical characteristics of guava fruit. J Food Process Preserv 40, 657-666.

17. Sun $\mathrm{T}, \mathrm{Xu} \mathrm{Z}$, Wu CT, Janes M, Prinyawiwatkul W, No HK (2007) Antioxidant activities of different colored sweet bell peppers (Capsicum annuum L.). J Food Sci 72, 98-102.

18. Brand-Williams W, Cuvelier M-E, Berset C (1995) Use of a free radical method to evaluate antioxidant activity. LWT-Food Sci Technol 28, 25-30.

19. Rousseau B (2004) Sensory evaluation techniques. In: Handbook of Food Analysis: Physical Characterization and Nutrient Analysis, 2nd edn, CRC Press, pp 21-37.

20. Steel RGD, Torrie JH, Others (1980) Principles and Procedures of Statistics, a Biometrical Approach, McGraw-Hill Kogakusha.

21. Freed R, Eisensmith SP, Goetz S, Reicosky D, Smail VW, Wolberg P (1991) User's guide to MSTAT-C: A software program for the design, management, and analysis of agronomic research experiments. Michigan State Univ East Lancing, MI.

22. Del Caro A, Piga A (2008) Polyphenol composition of peel and pulp of two Italian fresh fig fruits cultivars (Ficus carica L.). Eur Food Res Technol 226, 715-719.

23. Ramulu P, Rao PU (2003) Total, insoluble and soluble dietary fiber contents of Indian fruits. J Food Compos Anal 16, 677-685.

24. Slatnar A, Klancar U, Stampar F, Veberic R (2011) Effect of drying of figs (Ficus carica L.) on the contents of sugars, organic acids, and phenolic compounds. $J$ Agric Food Chem 59, 11696-11702.

25. Thonta GT, Patil VK (1988) Studies on drying of fig fruits. Indian Food Pack 42, 94-99.

26. Pawar SG, Kulkarni DN, Shere DM, Kulkarni KD, Patil VK (1992) Effect of pretreatments on chemical composition and drying rates of solar dried figs. Indian Food Pack 46, 39-44.

27. Dennison DB, Kirk JR (1982) Effect of trace mineral fortification on the storage stability of ascorbic acid in a dehydrated model food system. $J$ Food Sci 47, 1198-1200.

28. Eison-Perchonok MH, Downes TW (1982) Kinetics of ascorbic acid autoxidation as a function of dissolved oxygen concentration and temperature. $J$ Food Sci 47, 765-767.

29. Lund D (1988) Effects of heat processing on nutrients. In: Karmas E, Harris RS (eds) Nutritional Evaluation of Food Processing, Springer, Dordrecht, pp 319-354.

30. Ryley J, Kajda P (1994) Vitamins in thermal processing. Food Chem 49, 119-129.

31. Mir MA, Hussain PR, Fouzia S, Rather AH (2009) Effect of sulphiting and drying methods on physicochemical and sensorial quality of dried apricots during ambient storage. Int $J$ Food Sci Technol 44, 1157-1166.

32. García-Martínez E, Igual M, Martín-Esparza ME, Martínez-Navarrete N (2013) Assessment of the bioactive compounds, color, and mechanical properties of apricots as affected by drying treatment. Food Bioprocess Technol 6, 3247-3255.

33. Hollman PCH, Hertog MGL, Katan MB (1996) Analysis and health effects of flavonoids. Food Chem 57, 43-46.

34. Dueñas M, Pérez-Alonso JJ, Santos-Buelga C, Escribano-Bailón T (2008) Anthocyanin composition in fig (Ficus carica L.). J Food Compos Anal 21, 107-115.

35. Patthamakanokporn O, Puwastien P, Nitithamyong A, Sirichakwal PP (2008) Changes of antioxidant activity and total phenolic compounds during storage of selected fruits. J Food Compos Anal 21, 241-248.

36. Nadheesha MKF, Bamunuarachchi A, Edirisinghe E, Weerasinghe W (2007) Studies on antioxidant activity of Indian gooseberry fruit and seed. $J$ Sci Univ Kelaniya 3, 83-92.

37. Puoci F, Iemma F, Spizzirri UG, Restuccia D, Pezzi V, Sirianni R, Manganaro L, Curcio M, et al (2011) Antioxidant activity of a Mediterranean food product: fig syrup. Nutrients 3, 317-329.

38. Ishaq S, Rathore HA, Masud T, Ali S (2009) Influence of post harvest calcium chloride application, ethylene absorbent and modified atmosphere on quality characteristics and shelf life of apricot (Prunus armeniaca L.) fruit during storage. Pakistan J Nutr 8, 861-865.

39. Ehabe EE, Eyabi GDE, Numfor FA (2006) Effect of sugar and $\mathrm{NaCl}$ soaking treatments on the quality of sweet banana figs. J Food Eng 76, 573-578. 


\section{Appendix A. Supplementary data}

Table S1 Effect of different treatments on color (sensory properties) of dried fig during ambient storage.

\begin{tabular}{|c|c|c|c|c|c|c|c|c|}
\hline \multirow{2}{*}{ Treat } & \multicolumn{7}{|c|}{ Storage period (days) } & \multirow{2}{*}{ Mean \pm SD } \\
\hline & 0 & 30 & 60 & 90 & 120 & 150 & 180 & \\
\hline$T_{0}$ & $8.20 \pm 0.60^{\mathrm{a}}$ & $6.20 \pm 0.50^{c-f}$ & $5.40 \pm 0.90^{f-j}$ & $4.60 \pm 0.20^{\mathrm{jk}}$ & $2.80 \pm 0.10^{1}$ & $1.60 \pm 0.20^{\mathrm{m}}$ & $1.20 \pm 0.20^{\mathrm{m}}$ & $4.29 \pm 2.56^{\mathrm{e}}$ \\
\hline$T_{1}$ & $8.20 \pm 0.70^{\mathrm{a}}$ & $6.80 \pm 0.70^{\mathrm{bcd}}$ & $7.20 \pm 0.40^{\mathrm{b}}$ & $6.80 \pm 0.30^{\mathrm{bcd}}$ & $6.40 \pm 0.35^{\mathrm{b}-\mathrm{e}}$ & $6.20 \pm 0.40^{\mathrm{c}-\mathrm{f}}$ & $6.00 \pm 0.40^{\mathrm{d}-\mathrm{g}}$ & $6.80 \pm 0.74^{\mathrm{a}}$ \\
\hline$T_{2}$ & $8.40 \pm 0.40^{\mathrm{a}}$ & $5.40 \pm 0.40^{f-j}$ & $5.40 \pm 0.60^{f-j}$ & $5.60 \pm 0.40^{\mathrm{e}-\mathrm{i}}$ & $5.60 \pm 0.40^{\mathrm{e}-\mathrm{i}}$ & $5.00 \pm 0.50^{\mathrm{h}-\mathrm{k}}$ & $4.40 \pm 0.50^{\mathrm{k}}$ & $5.69 \pm 1.27^{\mathrm{d}}$ \\
\hline$T_{3}$ & $8.40 \pm 1.10^{\mathrm{a}}$ & $6.40 \pm 0.60^{\mathrm{b}-\mathrm{e}}$ & $6.40 \pm 0.70^{\mathrm{b}-\mathrm{e}}$ & $6.20 \pm 0.50^{\mathrm{c}-\mathrm{f}}$ & $6.20 \pm 0.70^{c-f}$ & $5.80 \pm 0.60^{\mathrm{e}-\mathrm{h}}$ & $5.40 \pm 0.70^{\mathrm{f}-\mathrm{j}}$ & $6.40 \pm 0.95^{\mathrm{b}}$ \\
\hline$T_{4}$ & $8.40 \pm 1.20^{\mathrm{a}}$ & $7.00 \pm 0.70^{\mathrm{bc}}$ & $6.80 \pm 0.80^{\mathrm{bcd}}$ & $6.80 \pm 0.70^{\mathrm{bcd}}$ & $6.20 \pm 0.17^{\mathrm{c}-\mathrm{f}}$ & $6.20 \pm 0.70^{\mathrm{c}-\mathrm{f}}$ & $6.00 \pm 0.60^{\mathrm{d}-\mathrm{g}}$ & $6.77 \pm 0.81^{\mathrm{a}}$ \\
\hline$T_{5}$ & $8.20 \pm 0.30^{\mathrm{a}}$ & $7.20 \pm 0.80^{\mathrm{b}}$ & $6.80 \pm 0.70^{\mathrm{bcd}}$ & $7.00 \pm 0.80^{\mathrm{bc}}$ & $6.80 \pm 0.50^{\mathrm{bcd}}$ & $6.40 \pm 0.40^{\mathrm{b}-\mathrm{e}}$ & $6.00 \pm 0.80^{\mathrm{d}-\mathrm{g}}$ & $6.91 \pm 0.69^{\mathrm{a}}$ \\
\hline$T_{6}$ & $8.40 \pm 0.80^{\mathrm{a}}$ & $5.40 \pm 0.20^{f-j}$ & $5.60 \pm 0.40^{\mathrm{e}-\mathrm{i}}$ & $6.00 \pm 0.40^{\mathrm{d}-\mathrm{g}}$ & $5.93 \pm 0.42^{\mathrm{d}-\mathrm{h}}$ & $5.20 \pm 0.30^{g-k}$ & $5.00 \pm 0.40^{\mathrm{h}-\mathrm{k}}$ & $5.93 \pm 1.15^{\mathrm{cd}}$ \\
\hline$T_{7}$ & $8.20 \pm 0.90^{\mathrm{a}}$ & $6.00 \pm 0.30^{\mathrm{d}-\mathrm{g}}$ & $5.80 \pm 0.50^{\mathrm{e}-\mathrm{h}}$ & $5.40 \pm 0.50^{\mathrm{f}-\mathrm{j}}$ & $5.60 \pm 0.60^{\mathrm{e}-\mathrm{i}}$ & $5.20 \pm 0.40^{g-k}$ & $4.80 \pm 0.80^{\mathrm{ijk}}$ & $5.86 \pm 1.11^{\mathrm{cd}}$ \\
\hline$T_{8}^{\prime}$ & $8.33 \pm 1.50^{\mathrm{a}}$ & $5.40 \pm 0.50^{f-j}$ & $6.40 \pm 0.60^{\mathrm{b}-\mathrm{e}}$ & $6.00 \pm 0.60^{\mathrm{d}-\mathrm{g}}$ & $5.80 \pm 0.40^{\mathrm{e}-\mathrm{h}}$ & $5.60 \pm 0.60^{\mathrm{e}-\mathrm{i}}$ & $5.60 \pm 0.40^{\mathrm{e}-\mathrm{i}}$ & $6.16 \pm 1.01^{b c}$ \\
\hline Mean & $8.30 \pm 0.10^{\mathrm{a}}$ & $6.20 \pm 0.71^{b}$ & $6.20 \pm 0.67^{b}$ & $6.04 \pm 0.77^{b}$ & $5.70 \pm 1.16^{c}$ & $5.24 \pm 1.46^{\mathrm{d}}$ & $4.93 \pm 1.51^{d}$ & \\
\hline
\end{tabular}

LSD: $\mathrm{T}=0.37, \mathrm{~S}=0.33, \mathrm{TS}=0.98$. Values are means of triplicate. The means within the row bearing same letter(s) are statistically significant at $p<0.05$.

Table S2 Effect of different treatments on flavor (sensory properties) of dried fig during ambient storage.

\begin{tabular}{|c|c|c|c|c|c|c|c|c|}
\hline \multirow{2}{*}{ Treat } & \multicolumn{7}{|c|}{ Storage period (days) } & \multirow{2}{*}{ Mean \pm SD } \\
\hline & 0 & 30 & 60 & 90 & 120 & 150 & 180 & \\
\hline$T_{0}$ & $8.40 \pm 0.70^{\mathrm{a}}$ & $7.60 \pm 0.50^{\mathrm{ab}}$ & $6.13 \pm 0.31^{\mathrm{d}-\mathrm{i}}$ & $4.60 \pm 0.40^{\mathrm{kl}}$ & $2.80 \pm 0.20^{\mathrm{m}}$ & $1.60 \pm 0.10^{\mathrm{n}}$ & $1.20 \pm 0.20^{\mathrm{n}}$ & $4.62 \pm 2.87^{\mathrm{d}}$ \\
\hline$T_{1}$ & $8.40 \pm 0.60^{a}$ & $7.20 \pm 0.60^{\mathrm{bc}}$ & $6.40 \pm 0.60^{c-g}$ & $6.20 \pm 0.40^{\mathrm{d}-\mathrm{h}}$ & $5.80 \pm 0.30^{f-j}$ & $5.60 \pm 0.40^{g-j}$ & $5.40 \pm 0.40^{\mathrm{h}-\mathrm{k}}$ & $6.43 \pm 1.05^{c}$ \\
\hline$T_{2}$ & $8.40 \pm 0.30^{a}$ & $8.20 \pm 0.70^{\mathrm{a}}$ & $7.20 \pm 0.70^{\mathrm{bc}}$ & $6.80 \pm 0.60^{\mathrm{b}-\mathrm{e}}$ & $6.40 \pm 0.40^{c-g}$ & $5.00 \pm 0.60^{\mathrm{jkl}}$ & $4.40 \pm 0.50^{1}$ & $6.63 \pm 1.51^{\mathrm{bc}}$ \\
\hline$T_{3}$ & $8.20 \pm 0.30^{\mathrm{a}}$ & $7.60 \pm 0.90^{\mathrm{ab}}$ & $7.20 \pm 0.40^{\mathrm{bc}}$ & $7.00 \pm 0.50^{\mathrm{bcd}}$ & $6.00 \pm 0.70^{\mathrm{e}-\mathrm{i}}$ & $5.60 \pm 0.70^{g-j}$ & $5.20 \pm 0.60^{\mathrm{i}-1}$ & $6.69 \pm 1.11^{\mathrm{bc}}$ \\
\hline$T_{4}$ & $8.20 \pm 0.80^{\mathrm{a}}$ & $7.60 \pm 0.40^{\mathrm{ab}}$ & $7.20 \pm 0.50^{\mathrm{bc}}$ & $6.60 \pm 0.70^{\mathrm{c}-\mathrm{f}}$ & $6.20 \pm 0.80^{\mathrm{d}-\mathrm{h}}$ & $6.00 \pm 0.80^{\mathrm{e}-\mathrm{i}}$ & $5.40 \pm 0.40^{\mathrm{h}-\mathrm{k}}$ & $6.74 \pm 0.98^{a b}$ \\
\hline$T_{5}^{4}$ & $8.40 \pm 0.60^{\mathrm{a}}$ & $8.20 \pm 0.50^{\mathrm{a}}$ & $7.60 \pm 0.60^{\mathrm{ab}}$ & $7.00 \pm 0.60^{\mathrm{bcd}}$ & $6.80 \pm 0.90^{\mathrm{b}-\mathrm{e}}$ & $6.00 \pm 0.50^{\mathrm{e}-\mathrm{i}}$ & $5.60 \pm 0.70^{g-j}$ & $7.09 \pm 1.06^{\mathrm{a}}$ \\
\hline$T_{6}$ & $8.40 \pm 0.70^{\mathrm{a}}$ & $7.60 \pm 0.60^{\mathrm{ab}}$ & $7.00 \pm 0.80^{\mathrm{bcd}}$ & $6.80 \pm 0.40^{\mathrm{b}-\mathrm{e}}$ & $5.60 \pm 0.60^{g-j}$ & $5.20 \pm 0.40^{\mathrm{i}-1}$ & $5.00 \pm 0.30^{\mathrm{jkl}}$ & $6.51 \pm 1.29^{c}$ \\
\hline$T_{7}$ & $8.40 \pm 1.00^{\mathrm{a}}$ & $7.60 \pm 0.70^{\mathrm{ab}}$ & $7.00 \pm 0.50^{\text {bcd }}$ & $6.80 \pm 0.70^{\mathrm{b}-\mathrm{e}}$ & $6.40 \pm 0.40^{c-g}$ & $6.20 \pm 0.40^{\mathrm{d}-\mathrm{h}}$ & $6.00 \pm 0.60^{\mathrm{e}-\mathrm{i}}$ & $6.91 \pm 0.85^{\mathrm{ab}}$ \\
\hline$T_{8}^{\prime}$ & $8.40 \pm 1.50^{\mathrm{a}}$ & $8.20 \pm 0.30^{\mathrm{a}}$ & $7.60 \pm 0.40^{\mathrm{ab}}$ & $7.20 \pm 0.60^{\mathrm{bc}}$ & $6.40 \pm 0.50^{c-g}$ & $6.20 \pm 0.70^{\mathrm{d}-\mathrm{h}}$ & $5.60 \pm 0.40^{g-j}$ & $7.09 \pm 1.06^{\mathrm{a}}$ \\
\hline Mean & $8.36 \pm 0.09^{a}$ & $7.76 \pm 0.36^{\mathrm{b}}$ & $7.04 \pm 0.49^{c}$ & $6.56 \pm 0.79^{d}$ & $5.82 \pm 1.19^{\mathrm{e}}$ & $5.27 \pm 1.44^{f}$ & $4.87 \pm 1.45^{g}$ & \\
\hline
\end{tabular}

LSD: $\mathrm{T}=0.36, \mathrm{~S}=0.32$, TS $=0.96$. Values are means of triplicate. The means within the row bearing same letter(s) are statistically significant at $p<0.05$.

Table S3 Effect of different treatments on taste (sensory properties) of dried fig during ambient storage.

\begin{tabular}{|c|c|c|c|c|c|c|c|c|}
\hline \multirow{2}{*}{ Treat } & \multicolumn{7}{|c|}{ Storage period (days) } & \multirow{2}{*}{ Mean \pm SD } \\
\hline & 0 & 30 & 60 & 90 & 120 & 150 & 180 & \\
\hline$T_{0}$ & $9.00 \pm 0.80^{a}$ & $8.40 \pm 0.40^{\mathrm{ab}}$ & $7.00 \pm 0.60^{\mathrm{d}-\mathrm{h}}$ & $6.80 \pm 0.60^{\mathrm{e}-\mathrm{h}}$ & $6.40 \pm 0.70^{f-j}$ & $6.00 \pm 0.70^{\mathrm{h}-\mathrm{k}}$ & $5.60 \pm 0.70^{\mathrm{ijk}}$ & $7.03 \pm 1.25^{\mathrm{a} b}$ \\
\hline$T_{1}$ & $8.20 \pm 0.60^{\mathrm{abc}}$ & $7.40 \pm 0.50^{\mathrm{b}-\mathrm{f}}$ & $7.20 \pm 0.40^{\mathrm{c}-\mathrm{g}}$ & $6.80 \pm 0.70^{\mathrm{e}-\mathrm{h}}$ & $6.80 \pm 0.80^{e-h}$ & $5.60 \pm 0.90^{\mathrm{ijk}}$ & $5.00 \pm 0.50^{\mathrm{k}}$ & $6.71 \pm 1.09^{\mathrm{b}}$ \\
\hline$T_{2}$ & $8.40 \pm 0.50^{\mathrm{ab}}$ & $8.20 \pm 0.40^{\mathrm{abc}}$ & $7.00 \pm 0.80^{\mathrm{h}}$ & $7.00 \pm 0.80^{\mathrm{d}-\mathrm{h}}$ & $6.80 \pm 0.90^{\mathrm{e}-\mathrm{h}}$ & $6.20 \pm 0.70^{g-j}$ & $6.00 \pm 0.90^{\mathrm{h}-\mathrm{k}}$ & $7.09 \pm 0.92^{\mathrm{ab}}$ \\
\hline$T_{3}$ & $8.40 \pm 0.80^{\mathrm{ab}}$ & $7.60 \pm 0.60^{\mathrm{b}-\mathrm{e}}$ & $7.60 \pm 0.60^{\mathrm{b}-\mathrm{e}}$ & $7.20 \pm 0.90^{c-g}$ & $6.80 \pm 0.60^{\mathrm{e}-h}$ & $6.40 \pm 0.80^{f-j}$ & $5.40 \pm 0.70^{\mathrm{jk}}$ & $7.05 \pm 0.97$ \\
\hline$T_{4}^{3}$ & $8.40 \pm 1.30^{\mathrm{ab}}$ & $8.20 \pm 0.70^{\mathrm{abc}}$ & $7.20 \pm 0.40^{c-g}$ & $7.20 \pm 0.60^{\mathrm{c}-\mathrm{g}}$ & $6.60 \pm 0.50^{\mathrm{e}-\mathrm{i}}$ & $6.20 \pm 0.40^{g-j}$ & $6.00 \pm 0.60^{h-k}$ & $7.11 \pm 0.93^{a b}$ \\
\hline$T_{5}$ & $8.20 \pm 0.80^{\mathrm{abc}}$ & $8.00 \pm 0.80^{\mathrm{a}-\mathrm{d}}$ & $7.60 \pm 0.50^{\mathrm{b}-\mathrm{e}}$ & $7.20 \pm 0.50^{c-g}$ & $6.80 \pm 0.40^{\mathrm{e}-\mathrm{h}}$ & $6.20 \pm 0.30^{g-j}$ & $6.00 \pm 0.70^{h-k}$ & $7.14 \pm 0.85^{a}$ \\
\hline$T_{6}$ & $8.40 \pm 0.60^{\mathrm{ab}}$ & $8.20 \pm 0.70^{\mathrm{abc}}$ & $7.60 \pm 0.35^{\mathrm{b}-\mathrm{e}}$ & $7.20 \pm 0.40^{c-g}$ & $7.00 \pm 0.60^{\mathrm{d}-\mathrm{h}}$ & $6.80 \pm 0.40^{\mathrm{e}-\mathrm{h}}$ & $6.20 \pm 0.60^{g-j}$ & $7.34 \pm 0.78^{a}$ \\
\hline$T_{7}$ & $8.40 \pm 1.10^{\mathrm{ab}}$ & $7.60 \pm 0.80^{\mathrm{b}-\mathrm{e}}$ & $7.60 \pm 0.45^{\text {be }}$ & $7.20 \pm 0.60^{c-g}$ & $7.00 \pm 0.50^{\mathrm{d}-\mathrm{h}}$ & $6.80 \pm 0.50^{\mathrm{e}-\mathrm{h}}$ & $6.20 \pm 0.80^{g-j}$ & $7.26 \pm 0.70^{\mathrm{a}}$ \\
\hline$T_{8}$ & $8.40 \pm 1.40^{\mathrm{ab}}$ & $8.00 \pm 0.90^{\mathrm{a}-\mathrm{d}}$ & $7.60 \pm 0.40^{\mathrm{b}-\mathrm{e}}$ & $7.20 \pm 0.70^{\mathrm{c}-\mathrm{g}}$ & $7.00 \pm 0.80^{\mathrm{d}-\mathrm{h}}$ & $6.40 \pm 0.60^{f-j}$ & $6.00 \pm 0.50^{\mathrm{h}-\mathrm{k}}$ & $7.23 \pm 0.85^{\mathrm{a}}$ \\
\hline Mean & $8.42 \pm 0.23^{\mathrm{a}}$ & $7.96 \pm 0.34^{b}$ & $7.38 \pm 0.27^{c}$ & $7.09 \pm 0.18^{\mathrm{cd}}$ & $6.80 \pm 0.20^{d}$ & $6.29 \pm 0.38^{\mathrm{e}}$ & $5.82 \pm 0.41^{\mathrm{f}}$ & \\
\hline
\end{tabular}

LSD: $\mathrm{T}=0.41, \mathrm{~S}=0.37, \mathrm{TS}=1.10$. Values are means of triplicate. The means within the row bearing same letter(s) are statistically significant at $p<0.05$. 